Internat. J. Math. \& Math. Sci.

Vol. 8 No. 3 (1985) 521-536

\title{
LOCALLY CONFORMAL SYMPLECTIC MANIFOLDS
}

\section{IZU VAISMAN}

\author{
Department of Mathematics
}

University of Haifa, Israel

(Received April 4, 1984)

ABSTRACT. A locally conformal symplectic (1.c.s.) manifold is a pair $\left(M^{2 n}, \Omega\right)$ where $M^{2 n}(n>1)$ is a connected differentiable manifold, and $\Omega$ a nondegenerate $2-$ form on $M$, such that $M=U_{\alpha} U_{\alpha}\left(U_{\alpha}\right.$ - open subsets), $\Omega / U_{\alpha}=e^{\sigma_{\alpha}} \Omega_{\alpha}, \sigma_{\alpha}: U_{\alpha} \rightarrow \mathbb{R}, d \Omega_{\alpha}=0$. Equivalently, $d \Omega=\omega \wedge \Omega$ for some closed 1 -form $\omega$. L.c.s. manifolds can be seen as generalized phase spaces of Hamiltonian dynamical systems since the form of the Hamilton equations is, in fact, preserved by homothetic canonical transformations. The paper discusses first Hamiltonian vector fields, and infinitesimal automorphisms (i.a.) on 1.c.s. manifolds. If $(M, \Omega)$ has an i.a. $X$ such that $\omega(X) \neq 0$, we say that $M$ is of the first kind and $\Omega$ assumes the particular form $\Omega=\mathrm{d} \theta-\omega \wedge \theta$. Such an $M$ is a 2-contact manifold with the structure forms $(\omega, \theta)$, and it has a vertical 2-dimensional foliation $V$. If $V$ is regular, we can give a fibration theorem which shows that $M$ is a $\mathrm{T}^{2}$-principal bundle over a symplectic manifold. Particularly, $V$ is regular for some homogeneous 1.c.s. manifolds, and this leads to a general construction of compact homogeneous 1.c.s. manifolds. Various related geometric results, including reductivity theorems for Lie algebras of $1 . a$. are also given. Most of the proofs are adaptations of corresponding proofs in symplectic and contact geometry. The paper ends with an Appendix which states an analogous fibration theorem in Riemannian geometry.

KEY WORDS AND PHRASES. Locally conformal symplectic manifold, s-contact manifold, Boothby-Wang fibration.

1980 MATHEMATICS SUBJECT CLASSIFICATION CODE. 53C15, $58 F 05$.

\section{INTRODUCTION.}

A symplectic manifold is a pair $\left(M^{2 n}, \Omega\right)$, where $M^{2 n}$ is an even dimensional differentiable manifold (all our manifolds are assumed $C^{\infty}$ and connected), and $\Omega$ is a closed nondegenerate 2-form on $M$. Such manifolds are very important since they provide a good geometric framework for Hamiltonian mechanics, and for other chapters of theoretical physics. If the 2 -form $\Omega$ is nondegenerate but not closed, $\left(\mathrm{M}^{2 \mathrm{n}}, \Omega\right)$ is an almost symplectic manifold, and this definition provides a class of geometrically 
interesting manifolds. In between, the locally conformal symplectic (1.c.s.) manifolds are defined as almost symplectic manifolds $M^{2 n}(n>1)$ which have an open covering $\left\{\mathrm{U}_{\alpha}\right\}_{\alpha \in A}$, and a system of functions $\sigma_{\alpha}: U_{\alpha} \rightarrow \mathbb{R}$ such that $\mathrm{d}\left(\mathrm{e}^{-\sigma_{\alpha}} \Omega\right)=0$. Equivalently, $\mathrm{d} \sigma_{\alpha}$ glue up to a closed 1 -form $\omega$, and

$$
\mathrm{d} \Omega=\omega \wedge \Omega \text {. }
$$

Formula (1.1) was established by H.C. Lee [ 1 ], and we call $\omega$ the Lee form; it is well defined, and $d \omega=0$. Clearly, iff $\omega$ is exact, the manifold is globally conformal symplectic (g.c.s.).

We refer the reader to $[2],[3],[4]$ and [5] for the first properties and examples of $1 . \mathrm{c.s} . m a n i f o l d s$, that also provide a geometric motivation for the study of this class of manifolds. But, let us also point out a physics motivation. Indeed, let us look at a dynamical system with $n$ degrees of freedom. Then its phase space can be seen as a $2 n$-dimensional differentiable manifold $M$, and the dynamics consists of the orbits of a well defined vector field $X$. Every point of $M$ has an open neighbourhood $U_{\alpha}$ with the local coordinates $\left(q_{(\alpha)}^{i}, p_{j}^{(\alpha)}\right)(i, j=1, \ldots, n)$ given by positions and momenta, and there is a Hamiltonian function $H_{(\alpha)}\left(q_{(\alpha)}^{i}, p_{j}^{(\alpha)}\right)$ such that the orbits are defined by the Hamilton equations

$$
\frac{\mathrm{dq}_{(\alpha)}^{\mathrm{i}}(\alpha)}{\mathrm{dt}}=\frac{\partial \mathrm{H}_{(\alpha)}}{\partial \mathrm{p}_{i}^{(\alpha)}}, \quad \frac{\mathrm{dp}_{i}^{(\alpha)}}{\mathrm{dt}}=-\frac{\partial \mathrm{H}_{(\alpha)}}{\partial \mathrm{q}_{(\alpha)}^{i}} .
$$

The well known symplectic interpretation [6] tells us that $X$ is precisely the Hamiltonian field of $\mathrm{H}_{(\alpha)}$ with respect to the symplectic form $\Omega_{(\alpha)}=\sum_{i=1}^{n} \mathrm{dq}{ }_{(\alpha)}^{i} \wedge d p_{i}^{(\alpha)}$. Now the usual continuation of this interpretation consists in asking the local forms $\Omega_{(\alpha)}$ and local functions $\mathrm{H}_{(\alpha)}$ to glue up to a global symplectic form $\Omega$, and a global Hamiltonian $H$. But this is not compulsory since the only global entity is $X$, and we must only ask that the transition functions

$$
q_{(\beta)}^{i}=q_{(\beta)}^{i}\left(q_{(\alpha)}^{k}, p_{h}^{(\alpha)}\right), \quad p_{i}^{(\beta)}=p_{i}^{(\beta)}\left(q_{(\alpha)}^{k}, p_{h}^{(\alpha)}\right)
$$

preserve the form of the Hamilton equations (1.2). This happens not only if (1.3) implies $\Omega_{\beta}=\Omega_{\alpha}$ (i.e., (1.3) are canonical transformations), but also if (1.3) implies $\Omega_{\beta}=\lambda_{B \alpha} \Omega_{\alpha}, \lambda_{B \alpha}=$ const. $\neq 0$ (i.e., (1.3) are homothetic canonical transformations) if we take $\mathrm{H}_{(\beta)}=\lambda_{\beta \alpha} \mathrm{H}_{(\alpha)}$. In other words, we get the Hamiltonian dynamics if the geometric structure of the phase space is defined by an open covering $\left\{U_{\alpha}\right\} \in_{A}$, and a corresponding system of local symplectic forms $\Omega_{\alpha}$, such that over $\mathrm{U}_{\alpha} \cap_{U_{\beta}} \neq \phi$ one has

$$
\Omega_{B}=\lambda_{B \alpha} \Omega_{\alpha}, \lambda_{B \alpha}=\text { const. . }
$$

In this case, we get easily from (1.4) the cocycle condition

$$
\lambda_{B \alpha} \lambda_{\alpha \gamma}=\lambda_{\beta \gamma} \text {, }
$$

hence we have a basic line bundle $L$ on $M$, and instead of a Hamiltonian function we have a Hamiltonian cross-section of $L$ (a "twisted Hamiltonian").

It is well known that the cocycle condition (1.5) implies

$$
\lambda_{B \alpha}=e^{\sigma_{\alpha}} / e^{\sigma_{\beta}}
$$

for some functions $\sigma_{\alpha}: U_{\alpha} \rightarrow \mathbb{R}$ defined up to a term $f / U_{\alpha}(f: M \rightarrow \mathbb{R})$, and then 
shows that

$$
\Omega=e^{\sigma_{\alpha}} \Omega_{\alpha}
$$

is a global non-degenerate 2 -form on $M$ defined up to a global factor. Hence $(M, \Omega)$ is an l.c.s. manifold.

Therefore, the 1.c.s. manifolds are natural phase spaces of Hamiltonian dynamical systems, more general then the symplectic manifolds, and this is the announced motivation.

Finally, we indicate that the 1.c.s. manifolds play an important role in the recent works of A. Lichnerowicz [?]

In this paper, we do not intend to discuss problems of mechanics or physics, but some problems concerning the differential geometrical structure of the 1.c.s. manifolds. In Section 2, we discuss infinitesimal automorphisms (i.a.) of an 1.c.s. structure $\Omega$. If there is an i.a. $X$ such that $\omega(X) \neq 0$, the manifold $(M, \Omega)$ is called of the first kind, and $s$ has a particular form, while $M$ becomes a 2-contact manifold [12]. This happens necessarily if $(M, \Omega)$ is homogeneous nonsymplectic. In Section 3, we define regular 1.c.s. manifolds, and give a corresponding Boothby-Wang fibration theorem [8], [ 9]. We also deduce that a homogeneous 1.c.s. manifold with an invariant $i . a . X$ such that $\omega(X) \neq 0$ is regular. In Section 4, we discuss compact homogeneous 1.c.s. manifolds, and show a method for constructing such manifolds as $T^{2}$ (torus)-bundles over compact homogeneous simply connected Hodge manifolds by applying the results of $[10]$ for contact manifolds. We also apply the method of [11] in order to derive some reductivity results for Lie algebras of $1 . a$. of 1.c.s. structures: In each section we also give various other related results. Most of the proofs are adaptations of corresponding proofs in symplectic and contact geometry. The paper closes with an Appendix where we give a Riemannian analogon of the BoothbyWang fibration theorem.

This text is a part of a series of lectures on Boothby-Wang fibration theorems given by the author at the Istituto Matematico del Politecnico di Torino (Italy), under the invitation of the Italian Consiglio Nazionale delle Ricerche (C.N.R.). I should like to express here my thanks to the CNR of Italy and to my hosts in Torino, particularly prof. F. Tricerri.

2. HAMILTONIAN FIELDS. INFINITESIMAL AUTOMORPHISMS.

Let $\left(M^{2 n}, \Omega\right)$ be an 1.c.s. manifold with the Lee form $w$, such that (1.1) holds (and $d \omega=0$ ). Then, we also have the characteristic vector field $A$ defined by $i(A) \Omega=\omega$, and it is easy to get

$$
i(A) \omega=0, L_{A} \omega=0, L_{A} \Omega=0 \text {. }
$$

Let $C^{\infty}(M)$ denote the associative algebra of $C^{\infty}$-functions on $M$, and $f: M \rightarrow \mathbb{R}$ be one such function. As in Section 1 , there is a well defined line bundle $L$ on $M$, and $f$ has ${ }^{a}$ well defined associated cross section $f^{L}$ of $L$ given by the local functions $f_{\alpha}=$ $=\mathrm{e}^{-\sigma_{\alpha}}$. Then, the usual symplectic Hamiltonian formalism $[6]$ provides us with the local fields $\mathrm{x}_{\mathrm{f}_{\alpha}}$ given by

$$
i\left(x_{f_{\alpha}}\right) \Omega_{\alpha}=\mathrm{df}_{\alpha}
$$

But (2.2) is equivalent to $i\left(x_{f_{\alpha}}\right) \Omega=d f-f \omega$, which shows that the local fields $x_{f_{\alpha}}$ glue up to a global vector field $x_{f}$ defined by

$$
i\left(x_{f}\right) \Omega=d f-f \omega .
$$


$\mathrm{X}_{\mathrm{f}}$ will be called the Hamiltonian vector field of $f$ with respect to the 1.c.s. form $\because$. Clearly, $x_{f}$ defines the dynamics of the local Hamiltonians $f_{\alpha}$ as described in Section 1 .

Using these fields, we define now the Poisson bracket

$$
\left\{f, g^{\prime}=\Omega\left(x_{g}, x_{f}\right)=x_{f} g-g \omega\left(x_{f}\right)=-x_{g} f+f \omega\left(x_{g}\right)=e^{\sigma \alpha_{\{f}}, g_{\alpha} \beta_{\alpha} .\right.
$$

The last expression of (2.4) shows that $P(M)=\left(C^{\infty}(M),\{.\},\right)$ is a Lie algebra (called the Poisson-Lie algebra of $(M, \Omega))$, and that one has

$$
x_{\{f, g\}}=\left[X_{f}, x_{g}\right]
$$

or, equivalently, the mapping $H: P(M) \rightarrow x(M)$ (where $x(M)$ is the Lie algebra of the vector fields of $M$ ) given by $f \rightarrow X_{f}$ is a Lie algebra homomorphism.

The following fact is rather interesting.

PROPOSITION 2.1 Let $(M, \Omega)$ be a (connected:) 1.c.s. manifold that is not g.c.s. Then $H$ is a monomorphism.

PROOF. By (2.3), $X_{f}=0$ means $d f-f \omega=0$, and $f$ cannot be nowhere zero since otherwise $\omega$ would be exact, and $M$ would be g.c.s. Hence, let $f\left(x_{0}\right)=0$ for $x_{0} \in M$, and put $\omega=-(d \tau / \tau)(\tau \neq 0)$ on some open connected neighbourhood of $x_{0} \cdot$ Then $d f-f \omega=0$ gives $d(f \tau)=0$, whence $f \tau=$ const., and $f \equiv 0$ on that neighbourhood. Now, for an arbitrary $x \in M$, one can build a chain of open connected neighbourhoods $U_{1}, \ldots, U_{n}$ such that $x_{0} \in U_{1}, x \in U_{n}, U_{i} \cap U_{i+1} \neq \phi\left(i=1, \ldots, r_{1}-1\right)$, and $\omega / U_{i}$ is exact $(i=1, \ldots, n)$. Then $f=0$ propagates along this chain from $x_{0}$ to $x^{i}$. Therefore, $x_{f}=0$ implies $f=0$. Q.E.D.

REMARK. This result is not true on g.c.s. manifolds.

Furthermore, it follows from (2.3) that any Hamiltonian field satisfies

$$
\mathrm{L}_{\mathrm{X}} \Omega=\omega\left(\mathrm{X}_{\mathrm{f}}\right) \Omega
$$

hence, generally and unlike in the symplectic case, $X_{f}$ is not an infinitesimal automorphism (i.a.) of $(M, \Omega)$. Of course, the latter are defined by $L_{X} \Omega=0$, and form a bracket-Lie algebra $x_{\Omega}(M)$. We do have $x_{f} \in x_{\Omega}(M)$ iff $\omega\left(x_{f}\right)=0$ or, equivalently in view of (2.1) and (2.3), Af $=0$. Vector fields $X$ such that $\omega(X)=0$ will be called

horizontal fields.

Now, let us refer to an arbitrary $x \in x_{\Omega}(M)$. Then we have $L_{x} \Omega=0$ and, by (1.1), $\mathrm{L}_{X} \omega=0$ as wel1. The later condition implies $\omega(X)=$ const. Particularly, if $X, Y \in X_{\Omega}(M)$, then $\omega(X)=$ const., $\omega(Y)=$ const., and $d \omega(X, Y)=0$ yield $\omega([X, Y])=0$. Hence, the application $\ell: X_{\Omega}(M) \rightarrow \mathbb{R}$ defined by $\ell(X)=\omega(X)$ is a Lie algebra homomorphism for the commutative Lie algebra structure of $\mathbb{R}$. We call $\ell$ the Lee homomorphism of $x_{\Omega}(M)$. The kernel ker $\ell$ is the Lie algebra of the horizontal elements of $x_{\Omega}(M)$, denoted $x_{\Omega}^{\text {hor }}(M)$. The i.a. $X \in X_{\Omega}(M)$ with $\ell(X) \neq 0$ will be called transversal i.a. (t.1.a.), and we shall say that the 1.c.s. manifold $M$ is of the first kind if it has t.1.a. Otherwis $\epsilon$, $M$ is of the second kind, and the Lee homomorphism is trivial. If $\omega$ has vanishing points, $M$ is necessarily of the second kind. Hence, if $M$ is of the first kind $\omega \neq 0$ everywhere, and, if $M$ is compact, $M$ has a vanishing Euler-Poincaré characteristic. If $(M, \Omega)$ is of the first kind, and $f: M \rightarrow I R$ is a function such that $d f / x_{0}=\omega\left(x_{0}\right)$, then $\left(M, e^{-f} \Omega\right)$ has the Lee form $\omega$-df with a vanishing point, and it is ..c.s. of the second kird. Clearly, if $M$ is 
of the first kind $\ell$ is onto, and we have the following exact sequence of Lie algebras

$$
0 \rightarrow x_{\Omega}^{\text {hor }}(\mathrm{M}) \stackrel{\subseteq}{=} x_{\Omega}(\mathrm{u}) \stackrel{\ell}{\rightarrow} \mathbb{R} \rightarrow 0 \text {. }
$$

It turns out that we can obtain much more information about the 1.c.s. ruanifolds of the first kind. Indeed, let us fix an element $B \in e^{-1}(1) \subset x_{\Omega}(M)$, and call $B$ the basic t.i.a. of $(M, \Omega)$. Then, every $Y \in X_{\Omega}(M)$ has a unique decomposition

$$
\mathrm{Y}=\mathrm{X}+\ell(\mathrm{Y}) \mathrm{B}, \quad \mathrm{X} \in \mathrm{X}_{\Omega}^{\text {hor }}(\mathrm{M}) \text {. }
$$

Now, put $\theta=-i(B) \Omega$ (hence $\theta(B)=0$ ), and write down $\mathrm{L}_{B} \Omega=0$ as $i(B) d \Omega+d i(B) \Omega=0$. This yields a particular expression for $\Omega$ namely

$$
\Omega=\mathrm{d} \theta-\omega \wedge \theta
$$

Furthermore, we have

$$
L_{B} \theta=-L_{B} i(B) \Omega=-i(B) d i(B) \Omega=-i(B)\left(L_{B} \Omega-i(B) d \Omega\right)=0,
$$

vhence

$$
i(B) \mathrm{d} \theta=0
$$

and rank $d \theta<2 n$. But then (2.9) and $\Omega^{n} \neq 0$ yield

$$
\omega \wedge \theta \wedge(d \theta)^{n-1} \neq 0
$$

everywhere. This yields

PROPOSITION 2.2. A manifold $\mathrm{M}^{2 \mathrm{n}}$ admits an 1.c.s. structure of the first kind iff it admits two 1 -forms $\omega, \theta$ such that $d \omega=0$, rank $d \theta<2 n$, and (2.12) holds at every point of $M$.

PROOF. Above, we obtained $\omega, \theta$ from $\Omega$. Conversely, if $\omega, \theta$ are given, (2.9) yields an l.c.s. structure $\Omega$ with Lee form $\omega$. Then the equations

$$
\omega(B)=1, \quad \theta(B)=0, \quad i(B) d \theta=0
$$

define a unique vector field $B$ on $M$ (that also satisfies $i(B) \Omega=-\theta$ ) such that $\mathrm{L}_{\mathrm{B}} \theta=0, \mathrm{~L}_{\mathrm{B}} \omega=0$. Hence $\mathrm{B}$ is a (basic) t.i.a. 2.E.D.

of course, $\omega$ and $\theta$ define $\Omega$ uniquely, but $\Omega$ does not define uniquely $(\omega, \theta)$. Note also that

$$
\omega(A)=0, \quad \theta(A)=1, \quad i(A) d \theta=0
$$

define the characteristic vector field of $M(i(A) \Omega=\omega)$, and since exp (t $B)$ preserves $\Omega$ it also preserves $A$. This means $[B, A]=0$, and we obtain on $M$ the vertical foliation $V=\operatorname{span}\{A, B\}$, whose leaves are the orbits of a natural action of $\mathbb{R}^{2}$. In the next Section, we shall use $V$ in order to get more geometric information on $M$.

In connection with the above discussion, we shall also make the following complementary considerations. Formula (2.6) proves that a Hamiltonian field is a conformal infinitesimal transformation (c.i.t.) of $(M, \Omega)$. Generally, a vector field $X$ of $M$ is a c.i.t. if [Lf]

$$
\mathrm{L}_{\mathrm{X}} \Omega=\alpha_{\mathrm{X}} \Omega,
$$

where $\alpha_{x}$ is a function on $M$. The c.i.t. form a bracket Lie algebra to be denoted by $x_{\Omega}^{c}(M)$, and if besides (2.15) one also has $\mathrm{L}_{\mathrm{Y}} \Omega=\alpha_{\mathrm{y}} \Omega$ it follows

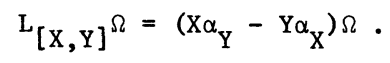

The Hamiltonian fields form a Lie subalgebra $\chi_{H a m}(M)$ of $\chi_{\Omega}^{c}(M)$. Now, if $X$ satis- 
fies (2.15) then, by differentiating this condition and since $n>1$, we get

$$
L_{X} \omega=d \alpha_{X},
$$

whence it follows that

$$
a_{X}=w(X)+k, k=\text { const. }
$$

If this $\alpha_{X}$ is used in (2.15), we see that (2.15) is equivalent to $L_{X} \Omega_{\alpha}=k \Omega_{\alpha}$, where $\Omega_{\alpha}$ are the local symplectic forms of the 1.c.s. structure. Hence $X$ is a c.i.t. iff it is an infinitesimal homothety of the forms $\Omega_{\alpha}$.

Furthermore, we can extend the Lee homomorphism to $l: x_{\Omega}^{c}(M) \rightarrow \mathbb{R}$ given by $\ell(X)=\omega(X)-\alpha_{X}=-k(k$ of $(2.18))$. If $X, Y \in X_{\Omega}^{c}(M)$, and we have (2.16), it follows $\ell([X, Y])=-d \omega(X, Y)=0$, hence the extended $\ell$ is also a Lie algebra homomorphism. Its kernel consists of fields $x$ such that $L_{x} \Omega_{\alpha}=0$, i.e., of locally Hamiltonian fields, and we denote $\operatorname{ker} \ell=X_{\mathrm{Ham}}^{\ell}(M) \supseteq X_{\mathrm{Ham}}(M)$. It is precisely the locally Hamiltonian fields that should be interesting in mechanics.

If the extended Lee homomorphism $\ell$ is nontrivial $1 . e$. , if there is a non-locally Hamiltonian field in $x_{\Omega}^{c}(M)$, we have the following exact sequence of lie algebras

$$
0 \rightarrow x_{\mathrm{Ham}}^{\ell}(\mathrm{M}) \stackrel{\subseteq}{\leftrightarrows} x_{\Omega}^{c}(M) \stackrel{\ell}{\rightarrow} \mathbb{R} \rightarrow 0 \text {, }
$$

and we shall say that $M$ has many c.i.t.

If this happens, let us fix an element $c \in l^{-1}(1)$, called a basic field, which gives uniquely for every $Y \in x_{\Omega}^{c}(M)$

$$
\mathrm{Y}=\mathrm{X}+\ell(\mathrm{Y}) \mathrm{C}, \quad \mathrm{X} \in \mathrm{X}_{\mathrm{Ham}}^{\ell}(\mathrm{M}) \text {. }
$$

Then, if $\gamma=-i(C) \Omega$, we have $L_{C} \Omega=i(C) d \Omega+d i(C) \Omega=\omega(C) \Omega+\omega \wedge \gamma-d \gamma=\alpha_{C} \Omega$, i.e., $\ell(C) \Omega+\omega \wedge \gamma-\mathrm{d} \gamma=0$ or equivalently

$$
\Omega=d \gamma-\omega \wedge \gamma \text {. }
$$

Hence, by comparing with (2.9), we see that an 1.c.s. manifold with many c.i.t. is a candidate of a manifold of the first kind. More precisely, let us note that the Lee homomorphism $\ell$ of $(2.19)$ is conformally invariant. Indeed, if $\tilde{\Omega}= \pm e^{\varphi_{\Omega}}$, then we get $\tilde{\omega}=\omega+\mathrm{d} \varphi$, and if $\mathrm{L}_{\mathrm{X}} \Omega=\alpha_{\mathrm{X}} \Omega$ we get $\mathrm{L}_{\mathrm{X}} \tilde{\Omega}=\tilde{\alpha}_{\mathrm{X}} \tilde{\Omega}$ with $\tilde{\alpha}_{\mathrm{X}}=\alpha_{\mathrm{x}}+\mathrm{x} \varphi$. Whence we obtain

$$
\tilde{\ell}(\mathrm{X})=\tilde{\omega}(\mathrm{X})-\tilde{\alpha}_{\mathrm{X}}=\omega(\mathrm{X})-\alpha_{\mathrm{X}}=\ell(\mathrm{X}) .
$$

Hence the existence of many c.i.t. is a conformally invariant property. Now, assume

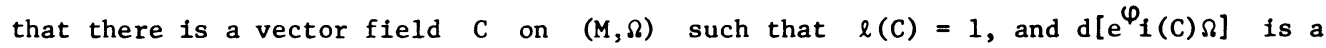
degenerate 2-form for some function $\varphi$ on $M$. Then Proposition 2.2 shows that $\left(M, e^{\varphi_{\Omega}}\right)$ is an 1.c.s. manifold of the first kind.

Let $(M, \Omega)$ be an 1.c.s. manifold of the first kind, and $B$ a basic t.i.a. Let $\chi_{\Omega}^{\text {hor }}(M, B)$ be the Lie subalgebra of $\chi_{\Omega}^{\text {hor }}(M)$ whose automorphisms also preserve $B$, i.e., $X \in \chi_{\Omega}^{\text {hor }}(M, B)$ iff $\omega(X)=0, L_{X} \Omega=0,[X, B]=0$. On the other hand denote by $C_{V}^{\infty}(M)$ the subset of $C^{\infty}(M)$ that consists of functions that are foliate with respect to the foliation $V$ or, equivalently, satisfy $A f=0, B f=0$, and remember the application $H(f)=X_{f}$. Then, we can prove

PROPOSITION 2.3. Let $M$ be an 1.c.s. manifold of the first kind which is not g.c.s. Then $C_{V}^{\infty}(M)$ is a Poisson-Lie subalgebra $P_{V}$ of $P(M)$, and $H$ sends iso- 
morphically $P_{V}$ onto $x_{\Omega}^{\text {hor }}(M, B)$.

PROOF. Let $f \in C_{V}^{\infty}(M)$, and $H(f)=X_{f}$. By (2.6) and the remark afterwards, since $A f=0, X_{f} \in X_{\Omega}^{\text {hor }}(M)$. Then, by (2.3) and (2.9) we get

$$
i\left(X_{f}\right) d \theta+\omega \cdot \theta\left(X_{f}\right)=d f-f \omega,
$$

which applied to $B$, and since $B_{f}=0$, implies

$$
\theta\left(\mathrm{X}_{\mathrm{f}}\right)=-\mathrm{f} \text {. }
$$

Now, (2.22) reduces to $i\left(X_{f}\right) d \theta=d f$, which with (2.23) implies $\operatorname{LX}_{f} \theta=0$, and because of $L_{X_{f}} \Omega=0$, we also have $\left[X_{f}, B\right]=0$. Hence $H$ sends $C_{V}^{\infty}(M)$ to $X_{\Omega}^{\text {hor }}(M, B)$, and it is injective because of (2.23).

Conversely, let $x \in x_{\Omega}^{\text {hor }}(M, B)$ (which implies $\omega(X)=0, L_{X} \theta=0$ ), and define $f=-\theta(X) \cdot$ Then

$$
\begin{aligned}
i(X) \Omega & =i(X)(d \theta-\omega \wedge \theta)=i(X) d \theta-\omega(X) \theta+\theta(X) \omega= \\
& =L_{X} \theta-d(\theta(X))-\omega(X) \theta+\theta(X) \omega=d f-f \omega
\end{aligned}
$$

i.e., $X=X_{f}$. Furthermore, as in the remark following (2.6), $\omega(X)=\omega\left(X_{f}\right)=0$ implies $A f=0$, and we also have $\left(L_{X} \theta\right)(B)=0=X(\theta(B))-\theta([X, B])=-\theta([X, B])$; $d \theta(B, X)=0=B(\theta(X))-X(\theta(B))-\theta([B, X])=B(\theta(X))$, i.e., $B f=0$. Hence $H$ is also a surjection for the sets of Proposition 2.3.

Finally, let $f, g \in C_{V}^{\infty}(M)$ and, therefore $x_{f}, x_{g} \in x_{\Omega}^{\text {hor }}(M, B)$. Then $\left[x_{f}, x_{g}\right]=$ $=x_{\{f, g\}} \in x_{\Omega}^{\text {hor }}(M, B)$ and, since by Proposition $2.1 H^{g}$ is injective, we must have $\{f, g\} \in C_{V}^{\infty}(M)$. Q.E.D.

We close this section by another simple but interesting result. An 1.c.s. manifold $(M, \Omega)$ is homogeneous if it admits a transitive lie group $G$ of $\Omega$-preserving diffeomorphisms. (In Section 4, we shall give a rather general construction of such manifolds.)

PROPOSITION 2.4. Let $(M, \Omega)$ be a homogeneous 1.c.s. manifold which is not symplectic. Then it is necessarily a manifold of the first kind.

PROOF. Remember that all our manifolds are connected. Then the homogenelty group $G$ may be assumed connected as well. Since $M$ is not symplectic and homogeneous, $\omega \neq 0$ everywhere, and $M$ is foliated by $\omega=0$. Let $p, q$ be points on different leaves of this foliation, and let $\gamma \in G$ such that $\gamma(p)=q$. Then we may write $\gamma=\exp \underline{x}_{1} \circ \ldots \circ \exp \underline{x}_{k}$ for some elements $\underline{x}_{\alpha}(\alpha=1, \ldots, k)$ of the Lie algebra $g$ of G. These elements have associated vector fields $x_{\alpha} \in x_{\Omega}(M)$, and we must have $\omega\left(x_{\alpha}\right) \neq 0$ for at least one index $\alpha$ since otherwise $\gamma$ acts along the leaves of $\omega=0$, and it cannot send $\mathrm{p}$ to $\mathrm{q}$. Q.E.D.

COROLLARY 2.5. A semisimple Lie group $G$ cannot act transitively on a nonsymplectic 1.c.s. manifold.

PROOF. Indeed, if $G$ is semisimple its Lie algebra $g$ is equal to the derived algebra $g^{\prime}$. But we know that any bracket of $i . a$. is horizontal. Hence $g$ would consist only of horizontal fields, which contradicts Proposition 2.4.

3. REGULAR L.C.S. MANIFOLDS.

In Section 2, we saw that an 1.c.s. manifold of the first kind has important foliations $U$. Inspired by a corresponding theory of contact manifolds [8], we shall define the regular 1.c.s. manifolds as 1.c.s. manifolds $M$ of the first kind for which 
it is possible to choose a basic t.i.a. B such that the corresponding vertical foliation $V=\operatorname{span}\{\mathrm{A}, \mathrm{B}\}$ is simple (regular), and the corresponding space of leaves $\mathrm{M} / \mathrm{V}=\mathrm{N}$ is a llausdorff differentiable manifold. Under these hypotheses, we may expect a fibration theorem, and, in fact, such a theorem was given for a more general structure [12], [ 9 ].

Namely, let $M^{2 n+s}$ be a differentiable manifold. An s-contact structure on $M$ consists of $s 1$-forms $\omega^{1}, \ldots, \omega^{s}$, and one 2 -form $\Omega$ of rank $2 n$ such that

$$
\begin{aligned}
& \omega^{1} \wedge \ldots \wedge \omega^{9} \wedge \Omega^{n} \neq 0 \quad \text { (everywhere), } \\
& \mathrm{d} \omega^{\mathrm{u}}=\alpha^{\mathrm{u}} \Omega\left(\alpha^{\mathrm{u}}=\text { const. } ; \mathrm{u}=1, \ldots, \mathrm{s}\right), \mathrm{d} \Omega=0 .
\end{aligned}
$$

If $\mathrm{n}>1, \alpha^{\mathrm{u}}=$ const. is a consequence of the other relations. If at least one of the $\alpha^{\mathrm{u}}$ is nonzero, the structure will be called of nonzero type. An s-contact structure defines a decomposition $\mathrm{TM}=\mathrm{C} \oplus V$ where $C$ (the horizontal bundle) is given by $\omega^{1}=0, \ldots, \omega^{s}=0$, and $V=\{\mathrm{X} / \mathrm{i}(\mathrm{X}) \Omega=0\}$ (the vertical bundle). Moreover, we have $s$ uniquely defined basic vertical vector fields $E_{u}(u=1, \ldots, s)$ given by

$$
i\left(E_{v}\right) \omega^{u}=\delta_{v}^{u}, i\left(E_{v}\right) \Omega=0(u, v=1, \ldots, s) .
$$

This relations imply

$$
\begin{aligned}
& \mathrm{L}_{E_{v}} \omega^{u}=i\left(E_{v}\right) d \omega^{u}=\alpha^{u} i\left(E_{v}\right) \Omega=0, \\
& L_{E_{v}} \Omega=0 \\
& i\left(\left[E_{u}, E_{v}\right]\right) \omega^{t}=L_{E_{u}} i\left(E_{v}\right) \omega^{t}-i\left(E_{v}\right) L_{E_{u}} \omega^{t}=0, \\
& i\left(\left[E_{u}, E_{v}\right]\right) \Omega=L_{E_{u}} i\left(E_{v}\right) \Omega-i\left(E_{v}\right) L_{E_{u}} \Omega=0,
\end{aligned}
$$

whence

$$
\left[E_{u}, E_{v}\right]=0(u, v,=1, \ldots, s) \text {. }
$$

Hence $V$ is a foliation of $M$ by the orbits of a natural action of $R^{s}$ on $M$. If this foliation is simple, and $N=M / V$ is Hausdorf $f$, we say that $M$ is a regular s-contact manifold, and the following result holds [12], [ 9 ].

PROPOSITION 3.1. Let $\left(\mathrm{M}^{2 \mathrm{n}+\mathrm{s}}, \omega^{\mathrm{u}}, \Omega\right)$ be a compact connected regular s-contact manifold, and $p: M^{2 n+s} \rightarrow N^{2 n}$ the corresponding submersion. Then $p$ is a principal $\mathrm{T}^{\mathrm{S}}$ (torus)-bundle, $\mathrm{N}$ is a symplectic manifold with the symplectic form $\Omega^{\prime}$ such that $\Omega=p^{*} \Omega^{\prime}$, and there are some constants $c^{u}$ such that $\left\{c^{u} \omega^{u}\right\}$ is a connection on $p$ with the curvature proportional to $\Omega^{\prime}$.

PROOF. The regularity hypothesis implies the regularity of the foliations by the orbits of $\mathrm{E}_{\mathrm{u}}$ for each $\mathrm{u}=1, \ldots, s$, whence these orbits must be embedded circles [8]. Then, for each $u=1, \ldots, s$, the period function $\mu_{E_{u}}(x)=\inf \left\{t / t>0, \exp \left(t E_{u}\right)(x)=x\right\}$ is a constant $c_{u} \neq 0$, in view of Tanno's theorem [13] applied to the pair $\left(E_{u}, \omega^{u}\right)$. Now, we see that the bracket commuting vector fields $\left(1 / c_{u}\right) E_{u}$ yield a free right action of $T^{s}=\mathbb{R}^{s} / \mathbb{Z}^{s}$ on $M$. Furthermore, let $U$ be a cubical flat regular coordinate neighbourhood for $(M, V)$ with the coordinates $\left(x^{a}, x^{u}\right)(a=1, \ldots, 2 n ; u=1, \ldots, s)$ such that $x^{a}=$ const. on the leaves of $V$. Put $U^{\prime}=p(U)$, and define $h: U^{\prime} \times T^{s} \rightarrow$ $\rightarrow \mathrm{p}^{-1}\left(\mathrm{U}^{\prime}\right)$ by

$$
h\left(\left(x^{a}\right),\left(t^{1}, \ldots, t^{s}\right)\right)=\exp \left(\frac{t_{1}}{c_{1}} E_{1}\right) \circ \ldots \circ \exp \left(\frac{t s}{c_{s}} E_{s}\right)\left(\sigma\left(x^{a}\right)\right),
$$

where $\sigma: U^{\prime} \rightarrow M$ is given by $\sigma\left(x^{a}\right)=\left(x^{a}, 0\right)$. Then $h$ is a diffeomorphism which 
gives a local trivialization for $p$ such that the right action of $T^{S}$ is right multiplication on the $T^{S}$-component in (3.7). This proves the principal bundle structure of p. All the other assertions of Proposition 3.1 are clean from (3.2), (3.3), (3.4). 2.E.D.

The following converse result is also clear.

PROPOSITION 3.2. Let $\left(N^{2 n}, \Omega^{\prime}\right)$ be a symplectic manifold, and $p: M^{2 n+s} \rightarrow N^{2 n}$ a $\mathrm{T}^{\mathrm{S}}$-principal bundle endowed with a connection $\left(\omega^{\mathrm{u}}\right)$ such that $\mathrm{d} \omega^{\mathrm{u}}=\alpha^{\mathrm{u}} \mathrm{p}^{*} \Omega^{\prime} \quad\left(\alpha^{\mathrm{u}}=\right.$ const.). Then $\left(\omega^{u}, p^{*} \Omega^{\prime}\right)$ is a regular s-contact structure on $M$.

Now, by Proposition 2.2, an 1.c.s. manifold $M$ admits associated 2-contact structures $(\omega, \theta, \mathrm{d} \theta)$ such that $\alpha^{1}=0, \alpha^{2}=1$, and conversely. Moreover, if $M$ is regular the associated 2-contact structure can be assumed regular, and we have

PROPOSITION 3.3. Let $M^{2 n}$ be a compact regular $1 . c . s$. manifold, and $p: M^{2 n} \rightarrow N^{2 n-2}$ be the corresponding submersion on the space of the leaves of a regular vertical foliation $V$ of $M$. Then $\mathrm{p}$ is a $\mathrm{T}^{2}$-principal fibre bundle over the symplectic manifold $N$. Conversely, if $P$ is such a principal bundle, and it is endowed with a connection $(\omega, \theta)$ such that $d \omega=0$, and $d \theta$ projects to the symplectic form of $N$, then $M$ is a regular 1.c.s. manifold.

Proposition 3.3 provides a construction method for regular 1.c.s. manifolds. In fact, it is easy to understand that $p$ can be obtained as a composition of principal fibrations: first, we can project $M$ onto the manifold $P$ of the orbits of $B$, and this will be a flat principal circle bundle over a regular contact manifold. Then project $\mathrm{P}$ onto $\mathrm{N}$ by the Boothby-Wang fibration [8] which is again a principal circle bundle. Particularly, the symplectic form of $\mathrm{N}$ must represent an integral cohomology class [8]. Conversely, the construction of $M$ will be realized in these two steps: construct $P$ like in [ 8], and then $M$ as a flat principal circle bundle over $\mathrm{P}$.

The results above are a straightforward generalization of the Boothby-Wang fibration theorem [8]. Moreover, many of the other results of the basic paper [7] can also be generalized straightforwardly to the present situation, and we shall indicate here this generalization.

PROPOSITION 3.4. Let $M$ be a regular compact 1.c.s. manifold. Then the group of the automorphisms of $M$ acts transitively on $M$.

PROOF. Let $U$ be a cubical flat regular coordinate neighbourhood of ( $M, V$ ) like in the proof of Proposition 3.1. Let $G$ be the automorphism group of $M$. Then, we see like in [8] that $G$ acts transitively along the slices of $\omega=0$ in $U$. But it also acts transitively on slices by the translations of the corresponding parameter. Hence $G$ acts transitively on $U$, and, because of connectedness, it also acts transitively on $M$. Q.E.D.

In [3] it is shown that, if $M$ is a compact connected 1.c.s., and $\omega \neq 0$ everywhere, then the group of its conformal transformations acts transitively on $M$.

Furthermore, an s-contact manifold $\left(M, \omega^{u}, \Omega\right)$ is called homogeneous if $M \approx G / K$, where $G$ is a Lie group of s-contact automorphisms which acts transitively and effectively on $M$, and $K$ is a closed subgroup of $G$. Hence, $\forall g \in G, g^{\star} \omega^{u}=\omega^{u}$, $\mathrm{g}^{*} \Omega=\Omega$ (the second relation follows from the first in the nonzero type case). Then 
one has

PROPOSITION 3.5. Let $\left(M=G / K, \omega^{u}, \Omega\right)$ be a homogeneous s-contact manifold of the nonzero type. Then $M$ is a regular s-contact manifold.

PROOF. [ 8 ] . The forms $\omega^{u}, \Omega$ lift to corresponding left-invariant forms $\widetilde{\omega}^{u}, \widetilde{\Omega}$ on $G$ which are ad K-invariant, and we shall look at the closed subgroup $\mathrm{H} \subset \mathrm{G}(\mathrm{H} \supset \mathrm{K})$ defined by

$$
H=\left\{h \in G /(\operatorname{adh})^{*} \tilde{\omega}=\widetilde{\omega}^{\mu},(\operatorname{adh})^{*} \widetilde{\Omega}=\widetilde{\Omega}\right\} .
$$

Then, if $g$ denotes the Lie algebra of $G, \underline{h}$ of $H$ and $\underline{k}$ of $K$, if we denote by $\underline{X}$ a generical element of $g=T_{e} G$ ( $e$ is the unit of $G$ ), and by $X$ the corresponding left invariant field of $G$, we get:

$$
\underline{\mathrm{h}}=\left\{\underline{\mathrm{X}} \in \underline{\mathrm{g}} / \mathrm{L}_{\mathrm{X}} \widetilde{\omega}^{\mathrm{u}}=0, \mathrm{~L}_{\mathrm{X}} \widetilde{\Omega}=0\right\} .
$$

Since everything in the above construction is left-invariant, if we use the definition of the lie derivative, we see that the conditions which define $\underline{x} \in \underline{h}$ are equivalent to

$$
\left(\mathrm{d} \widetilde{\omega}^{u}\right)(\underline{X}, \underline{Y})=0, \tilde{\Omega}_{e}(\underline{X},[\underline{Y}, \underline{z}])=0,
$$

where $\underline{\mathrm{Y}}, \underline{z}$ are arbitrary elements of $g$, and we also used $d \tilde{\Omega}=0$. Since $\alpha^{u} \neq 0$ for some index $u, L_{X} \widetilde{\Omega}=0$ follows from $L_{X} \widetilde{\omega}^{u}=0$, and the only remaining condition is the first condition (3.8), which is equivalent to $\widetilde{\Omega}_{e}(\underline{x}, \underline{Y})=0$. I.e., $\underline{h}=\left\{\underline{X} \in \underline{g} / \widetilde{\Omega}_{e}(\underline{X}, \underline{Y})=0\right\}$, and since rank $\widetilde{\Omega}=2 n[8]$, we get $\operatorname{dim} \underline{h}=\operatorname{dim} \underline{k}+s$. Furthermore, it is known that for a triple $G \supset H \supset K$ as above there is a natural diagram of locally trivial fibrations

$$
\text { of } \underset{G / H}{\stackrel{\pi}{\longrightarrow} G / K}
$$

where, particularly, $\rho$ has the structure group $H$ and the s-dimensional fibre $H / K$. Now, if $E_{u}$ are the basic vertical fields on $G / K$, there are (non-unique) left-invariant lifts $\widetilde{E}_{u}$ to $G$ which satisfy $i\left(\widetilde{E}_{j}\right) \widetilde{\Omega}=0$ so that $\widetilde{E}_{u}(e)$ are in h. It is easy to deduce from this that the tangent distribution of the leaves of $V$ on $G / K$ is precisely the vertical distribution of the fibration $\rho$. But then, by applying the Corollary on p. 28 of [14], it follows that the foliation $V$ is simple and its basis is a covering manifold of $G / H$ i.e., a Hausdorff manifold. 2.E.D.

REMARKS. 1) Just like in [8], we can see that the space of the leaves $(G / K) / V$ is $G /\left(H_{0} \cdot K\right)$, where $H_{0}$ is the connected component of $e$ in $H .2$ ) The proof of Proposition 3.5 also holds for all $\alpha^{\mathrm{u}}=0$ if $\mathrm{G}$ is semisimple but the same argument as for Corollary 2.5 shows that, if $\alpha^{\mathrm{u}}=0$ holds for at least one index $u, G$ cannot be semisimple.

COROLLARY 3.6. Let $(M, \Omega)$ be a homogeneous 1.c.s. manifold which admits an invariant t.i.a. Then $M$ is a regular 1.c.s. manifold.

The supplementary hypothesis is necessary in order to have a homogeneous associated 2-contact structure, and to apply to it Proposition 3.5. We notice that if $(M, \Omega)$ is a homogeneous nonsymplectic 1.c.s. manifold such that $M=G / K$, where $G$ is a reductive Lie group (particularly, $G$ is a compact group), then $M$ must have an invariant t.i.a. Indeed, as in the proof of Proposition 2.4 , there is an element $\underline{x} \in \underline{g}$ 
which generates a t.i.a. $X_{M}$. But then (2.7) yields an exact sequence of Lie algebras

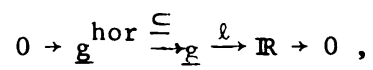

where $g^{\text {hor }}$ consists of elements of $g$ which yield horizontal fields on $M$, and since $g$ is reductive $g^{\text {hor }}$ has an ad $G$-invariant complement. 2.E.D. If $G$ is reductive (compact) we shall say that $M$ is of the reductive (compact) type.

4. COMPACT HOMOGENEOUS L.C.S. MANIFOLDS.

Like for the regularity property, we may expect to obtain information about compact homogeneous 1.c.s. manifolds from a discussion of compact honogeneous s-contact manifolds, and for the latter it is possible to extend in a rather straightforward manner the results established for contact manifolds in [10].

Let $\left(M=G / K, \omega^{u}, \Omega\right)$ be (here, and always in the sequel) a compact homogeneous s-contact manifold of nonzero type. Then $M$ is regular by Proposition 3.5, and there is a $T^{S}$-principal bundle $p: M \rightarrow N$ over the symplectic manifold (N, $\left.\Omega^{\prime}\right)$ with $\mathrm{p}^{*} \Omega^{\prime}=\Omega$. Obviously, $\mathrm{N}$ is also compact and symplectic homogeneous with the homogeneity group $G$ since $G$ preserves the whole structure of $M$, and, particularly, the vertical foliation $V$ (see Section 3 for notation). Now, recall that a homogeneous symplectic manifold with group $G$ is homogeneous strongly symplectic if for every $\underline{X} \in \underline{g}$ the field $X_{N}$ induced on $N$ is a Hamiltonian field. Then we have

PROPOSITION 4.1. The basis $N$ of the projection $\mathrm{p}: \mathrm{M} \rightarrow \mathrm{N}$ above is homogeneous strongly symplectic.

PROOF. [10]. Consider $\underline{X} \in \underline{g}$ and the induced fields $x_{M}$ on $M, x_{N}=p_{*} x_{M}$ on $N$, and denote, as usual, by $E_{u}(u=1, \ldots, s)$ the basic vertical vector fields of $M$, that are obviously G-invariant fields. Assume, for instance, $\alpha^{1} \neq 0$, whence $\Omega=\left(1 / \alpha^{1}\right) \mathrm{d} \omega^{1}$. Then

$$
E_{u}\left(\omega^{1}\left(X_{M}\right)\right)=L_{E_{u}} i_{X_{M}} \omega^{1}=i\left(\left[E_{u}, X_{M}\right]\right) \omega^{1}+i\left(X_{M}\right) L_{E_{u}} \omega^{1}=0
$$

$(u=1, \ldots, s)$, and $\omega^{1}\left(X_{M}\right)$ projects to a function $f_{\underline{X}}$ on $N$. Furthermore, since $\mathrm{L}_{\mathrm{M}}{ }^{1}=0$, we have

$$
p *\left(i_{X_{N}} \Omega^{\prime}\right)=i_{X_{M}} \Omega=\frac{1}{\alpha^{1}} i\left(X_{M}\right) d \omega^{1}=-\frac{1}{\alpha^{1}} d\left(\omega^{1}\left(X_{M}\right)\right)=-\frac{1}{\alpha^{1}} p * d f_{X},
$$

which yields $i_{X_{N}} \Omega^{\prime}=-\left(1 / \alpha^{1}\right) d_{X}$, and shows that $x_{N}$ is a Hamiltonian field. Q.E.D.

COROLLARY 4.2. The basis $N$ of the projection $P: M \rightarrow N$ described at the beginning of this section is a simply connected compact homogeneous symplectic manifold, and its symplectic form belongs to a Kähler metric that is homothetical to a Hodge metric. The Betti numbers $b_{2 h+1}(N)$ are zero.

Al1 this is gathered in Theorem 1 of $[10$, p. 341] on the basis of results of Bore1, Lichnerowicz and Milnor.

The above results give us the structure of compact homogeneous s-contact manifolds of the nonzero type as $\mathrm{T}^{\mathrm{S}}$-principal bundles over special homogeneous symplectic manifolds. Conversely, we have

PROPOSITION 4.3. Let $\mathrm{p}: \mathrm{M}^{2 \mathrm{n}+\mathrm{s}} \rightarrow \mathrm{N}^{2 \mathrm{n}}$ be a $\mathrm{T}^{\mathrm{s}}$-principal bundle, where $\mathrm{N}^{2 \mathrm{n}}$ is a compact simply connected homogeneous Hodge manifold with the Kähler form $\Omega^{\prime}$. Assume $\mathrm{p}$ has a connection $\left(\omega^{\mathrm{u}}\right)(\mathrm{u}=1, \ldots, \mathrm{s})$ such that $\mathrm{d} \omega^{\mathrm{u}}=\alpha^{\mathrm{u}} \mathrm{p}^{*} \Omega^{\prime}$, where not 
all $\alpha^{\mathrm{u}}=0$. Then $\left(\mathrm{M}, \omega^{\mathrm{u}}, \Omega=\mathrm{p} * \Omega^{\prime}\right)$ is a compact homogeneous s-contact manifold. PROOF. That $\left(\omega^{\mathrm{u}}, \Omega\right)$ is a regular s-contact structure is known by Proposition 3.2. The homogeneity of this structure will be proven like for the contact case in [ 10 ]. Namely, by results of Montgomery and Lichnerowicz as quoted in [10], we may assume $\mathrm{N}=\mathrm{G} / \mathrm{K}$ with $\mathrm{G}$ a compact and semisimple Lie group, and $\left(\mathrm{N}, \Omega^{\prime}\right)$ is a Hamiltonian space, i.e., there is a Lie algebra homomorphism $\varphi: g \rightarrow P(N)$ (the Poisson algebra) such that $\forall \underline{X} \in \underline{g}$ one has

$$
i\left(\mathrm{X}_{\mathrm{N}}\right) \Omega^{\prime}=\mathrm{d}(\varphi(\underline{\mathrm{X}}) \text {. }
$$

Now, take the Lie algebra $g \oplus \mathbb{R}^{S}$ with the zero bracket in $\mathbb{R}^{S}$, and define $\gamma: g \rightarrow \mathbb{R}^{S} \rightarrow x(M)$ by

$$
\gamma\left(\underline{X} \oplus\left(t^{u}\right)_{u=1, \ldots, s}\right)=\widetilde{X}_{N}-(\varphi(\underline{X}) \circ p) \underset{u=1}{\sum} \alpha^{u} E_{u}-\stackrel{\Sigma}{\Sigma}_{u=1}^{s} t^{u} E_{u},
$$

where $\tilde{\mathrm{x}}_{N}$ is the horizontal lift of $\mathrm{x}_{N}$ with respect to the connection $\left(\omega^{\mathrm{u}}\right), \alpha^{\mathrm{u}}$ are the constants which appear in Proposition 4.3, and $E_{u}$ are the basic vertical vector fields of the s-contact structure of $M$. Then, a computation similar to that of [10, p. 347] shows that $\gamma$ is a Lie algebra homomorphism and, therefore im $\gamma$ is a finite dimensional Lie subalgebra of $\chi(M)$. Accordingly, there is a connected Lie group $\vec{G}$ of left transformations of $M$, and we can see like in [10] that $\bar{G}$ acts transitively on $M$. (I.e., $\forall \mathrm{x}_{0} \in \mathrm{M}$ and $\mathrm{v} \in \mathrm{T}_{\mathrm{x}_{0}} \mathrm{M}$, if we decompose $v=$ horizontal $(v)+\sum_{u=1}^{S} \omega^{u}(v) E_{u}$, and if $\underline{x} \in g$ is such that $\tilde{X}_{N}\left(x_{0}\right)=$ horizontal (v), which is possible since $G$ is transitive on $N$, the field

$$
\mathrm{A}=\gamma\left[\underline{\mathrm{X}} \odot\left(-\varphi(\underline{\mathrm{X}}) \circ \mathrm{p}\left(\mathrm{x}_{0}\right) \alpha^{\mathrm{u}}-\omega^{\mathrm{u}}(\mathrm{v})\right)_{\mathrm{u}}=1, \ldots, \mathrm{s}\right]
$$

is such that $A\left(x_{0}\right)=v$. This implies the transitivity of $\overline{\mathbf{G}}$. )

Finally, we see again like in [10] that $L_{\gamma}\left(\underline{X} \oplus\left(t^{u}\right)\right)^{\omega^{u}}=0$, and $L_{\gamma}\left(\underline{X} \oplus\left(t^{u}\right)\right)^{\Omega=0}$ follows since the s-structure is of the nonzero type. This means that $\overline{\mathrm{G}}$ preserves the s-structure of $M$. Q.E.D.

From these results it follows:

COROLLARY 4.4. Let $(M, \Omega)$ be a compact homogeneous 1.c.s. manifold which admits an invariant t.i.a. Then $M$ has a $T^{2}$-principal bundle structure $p: M^{2 n} \rightarrow N^{2 n-2}$, where $\mathrm{N}$ is a simply connected compact homogeneous Hodge manifold. Conversely, every such bundle which is endowed with an adequate connection as in Proposition 4.3 is a compact homogeneous 1.c.s. manifold. If $M$ is as in the present Corollary, its first Betti number is $b_{1}(M)=1$.

Here, only the last assertion has to be justified, and it follows by first considering $M$ as a flat circle bundle over a contact manifold $P$, then fibering $P$ over $\mathrm{N}$ as a principal fibre bundle, and finally by applying twice the Gysin exact sequence theorem and using the fact that $b_{1}(N)=0$.

Now, let us consider again a homogeneous 1.c.s. manifold $(M=G / K, \Omega)$, let $g$ be the Lie algebra of $G$, and $\underline{g}^{\text {hor }}$ the subalgebra of those $\underline{X}$ of $g$ that $\omega\left(X_{M}\right)=0$. Accordingly, we have the exact sequence (3.10). The symplectic case suggests us to say that $M$ is strongly homogeneous if for every $\underline{x} \in \underline{g}^{\text {hor }}, X_{M}$ is the Hamiltonian field $x_{f}$ of a function $f \in P(M)$ with $A f=0$ (see Section 2). Similarly, $M$ is Hamiltonian 1.c.s. if a Lie algebra homomorphism $\varphi: \underline{g}^{\text {hor }} \rightarrow P(M)$ exists such that 
$\mathrm{A}(\mathrm{im} \varphi)=0$, and $\forall \underline{\mathrm{x}} \in \underline{\mathrm{g}}^{\text {hor }}$ one has $\mathrm{X}_{M}=\mathrm{X}_{\varphi(\underline{X})}$. If $M$ is not g.c.s., and it has a G-invariant t.i.a. then we have the Lie algebra isomorphism $H$ of Proposition 2.3, and $H^{-1}$ restricted to fields $X_{M}$ defined by $\underline{x} \in g^{\text {hor }}$ yields a homomorphism showing that $M$ is a Hamiltonian 1.c.s.

PROPOSITION 4.5. Let $(M=G / K, \Omega)$ be a compact strongly homogeneous 1.c.s. manifold which is not g.c.s., and assume $G$ is connected. Then the Lie algebra $g^{\text {hor }}$ is semisimple if $A \notin g$, and $\underline{g}$ hor $=\underline{s} \oplus \operatorname{span}\{A\}(\underline{s}-\operatorname{semisimple})$ if $A \in \underline{g}$. PROOF. [11]. Every $\underline{X} \in g^{\text {hor }}$ satisfies $X_{M}=X_{f(X)}$, Af $=0$, and in view of Proposition 2.1, $f$ is unique. Accordingly, we may define on $g$ hor an inner product

$$
\langle\underline{X}, \underline{Y}\rangle=\frac{1}{n !} \int_{M} f(\underline{X}) f(\underline{Y}) \Omega^{n},
$$

where $M$ is oriented such that $\Omega^{n}>0$, and if $\underline{z} \in \underline{g}^{\text {hor }}$ we have

$$
\begin{gathered}
\langle[\underline{Z}, \underline{X}], \underline{Y}\rangle+\langle\underline{X},[\underline{Z}, \underline{Y}]\rangle=\frac{1}{n !} \int_{M}[\{f(\underline{Z}), f(\underline{X})\} \cdot f(\underline{Y})+ \\
+f(\underline{X}) \cdot\{f(\underline{Z}), f(\underline{Y})\}] \Omega \Omega^{n}=\frac{1}{n !} \int_{M} Z_{M}(f(\underline{X}) \cdot f(\underline{Y})) \Omega^{n}=\frac{1}{n !} \int_{M} d\left(i(Z,) f(\underline{X}) f(\underline{Y}) \Omega^{n}\right)=0 .
\end{gathered}
$$

That is, the inner product $(4.3)$ is $\mathrm{Ad} \mathrm{g}^{\text {hor }}$-invariant, and we conclude that $\mathrm{g}^{\text {hor }}$ is a reductive Lie algebra, and that $\underline{g}^{\text {hor }}=\underline{s} \oplus \underline{c}$, where $\underline{s}$ is semisimple, and $\underline{c}$ is the centre of $\underline{g}^{\text {hor }}$.

Now, let us note that there is a connected subgroup $G^{\text {hor }} \subset \mathrm{G}$ whose Lie algebra is $g^{\text {hor }}$, and $G^{\text {hor }}$ acts transitively on every leaf $L$ of the foliation $\omega=0$. Indeed, if $p, q \in L$, a leaf of $\omega=0$, and $q=g(p), g \in G, g=\exp _{1}{ }^{\circ} \ldots \circ \exp \underline{x}_{h}$, $\underline{x}_{1}, \ldots, \underline{x}_{h} \in g$, then, generally, the situation is such that, for instance, $\underline{x}_{1} g^{\text {hor }}$, $\underline{x}_{2} \notin g^{\text {hor }}, \underline{x}_{3} \in \underline{g}^{\text {hor }}$, etc. But then $\exp \underline{x}_{1}$ sends the leaf $L$ to a leaf $L^{\prime}$, exp $\underline{x}_{2}$ sends $L^{\prime}$ to $L^{\prime \prime}, \exp \underline{X}_{3}$ preserves $L^{\prime \prime}$ etc., and we also must have some $\underline{X}_{u}$, $\underline{X}_{v}, \underline{X}_{w}$ such that their exponentials bring us back from L" (or whatever other leaf) to L. Since any bracket of $i . a$. is horizontal, if we exchange in $g$ the order of the exponentials such that $\underline{X}_{\mathbf{u}}, \underline{X}_{-}, \underline{x}_{w}$ come next to $\underline{X}_{1} \underline{X}_{2}$, this adds a factor in ${ }^{\text {hor }}$. Then, exp $\underline{x}_{1} \circ \exp \underline{x}_{2} \circ \exp \underline{x}_{u} \circ \exp \underline{x}_{v} \circ \exp \underline{X}_{w}$ preserves $L$, and is also in $G^{\text {hor }}$. Therefore, eventually, we get some $\gamma \in G^{\text {hor }}$ such that $\gamma(p)=q$. 2.E.D. This clearly implies that $\forall p \in L$, and $\xi_{p} \in T_{p} L$ there is an element $\underline{x} \in \underline{g}^{\text {inor }}$ such that $X_{M}(p)=\xi_{p}$.

Furthermore, let us look at the previous decomposition $\underline{g}^{\text {hor }}=\underline{s} \oplus \underline{c}$, and consider $\underline{X} \in \underline{c}$ i.e., $[\underline{X}, \underline{Y}]=0$ for all $\underline{Y} \in \underline{g}^{\text {hor }}$. This implies $X_{\{f(\underline{X}), f(\underline{Y})\}}=0$, hence $\{f(\underline{X}), f(\underline{Y})\}=-\Omega\left(X_{M}, Y_{M}\right)=0$. This equality together with the previous argument shows that $\left(i\left(x_{M}\right) \Omega\right)_{p}\left(\xi_{p}\right)=0, \forall p \in M$ and $\forall \xi_{p} \in T_{p} M$ such that $\omega_{p}\left(\xi_{p}\right)=0$, i.e., $i\left(x_{M}\right) \Omega=\lambda \omega$ for some function $\lambda$, and $x_{M}=\lambda$ A. 2.E.D.

Let us note the following Corollary which, as a matter of fact, follows also from the proof of Proposition 4.3.

COROLLARY 4.6 . Let $(M=G / K, \Omega)$ be a compact homogeneous 1.c.s. but not g.c.s. manifold with an invariant t.i.a.B. Then $M$ can be also represented as $\widetilde{G} / \widetilde{K}$ where the Lie algebra of $\tilde{G}$ is of the form $\tilde{g}=\underline{s} \oplus \mathbb{R}^{2}$, and $\underline{s}$ is a semisimple Lie algebra. PROOF. Consider the Lie algebra $\tilde{g}=\underline{g} \oplus \operatorname{span}\{\mathrm{A}\} \operatorname{span}\{\mathrm{B}\}$, Clearly, $\tilde{\mathbb{q}}^{\text {hor }}=$ $\underline{\mathrm{g}}^{\text {hor }}+\operatorname{span}\{\mathrm{A}\}$. By Proposition 4.5 , we have $\tilde{g}^{\text {hor }}=\underline{\mathbf{s}} \oplus \operatorname{span}\{\mathrm{A}\}$, and the result follows. 
Another related result is

COROLLARY 4.7. Let $(M=G / K, \Omega)$ be a compact strongly homogeneous 1.c.s. but not g.c.s. manifold such that $A$ doesn't represent an element of $g$. Then $M$ has a G-invariant transversal infinitesimal automorphism $B$.

PROOF. We know that the Lie algebra $g$ is not semisimple, and, therefore, it must have a nonzero abelian ideal $\underline{I}$. Then, $\underline{I} \cap \underline{g}^{\text {hor }}$ is a commutative ideal of $g^{\text {hor }}$ and since, under the hypothesis, $\underline{g}^{\text {hor }}$ is semisimple, $\underline{I} \cap g^{\text {hor }}=0$. Hence $\operatorname{dim} \underline{I}=1$, and $\underline{I}$ can be seen as generated by $\underline{B}$ where $B_{M}=B$ and $\omega(B)=1$. Since $\underline{I}$ is an ideal we have $[\underline{x}, \underline{B}]=\lambda \underline{B}, \forall \underline{x} \in \underline{g}$, and since $[\underline{x}, \underline{B}]$ has to be horizontal, $[\underline{x}, \underline{B}]=0$, and $\underline{B}$ is a central element in $\underline{g}$. But then $B$ is the requested $i . a$. 2.E.D.

\section{RIEMANNIAN MANIFOLDS}

In the present paper, we used the Boothby-Wang fibration technique of [8] in order to clarify the geometric structure of a regular 1.c.s. manifold. This is an interesting technique, and we should like to indicate here a different application of it. This section is not on 1.c.s. manifolds but on Riemannian manifolds.

Let $\mathrm{M}^{\mathrm{m}}$ be a compact connected Riemannian manifold with the metric $\mathrm{g}$. Let us assume that there is given an action of the additive group $\mathbb{R}^{S}$ on $M^{m}$ by isometries of $\mathrm{g}$ all of whose orbits are s-dimensional. Then, the orbits of this action define on $M$ a foliation $V$ (called the vertical foliation) whose leaves are s-dimensional submanifolds tangent to some independent commuting vector fields $E_{u}(u=1, \ldots, s)$ provided by the natural basis of $\mathbb{R}^{S}$. Clearly, we have $\mathrm{L}_{\mathrm{E}_{\mathrm{u}}} \mathrm{g}=0$. If $V$ is a simple foliation whose space of leaves is Hausdorff, we say that the action of $\mathbb{R}^{S}$ on $M$ is regular.

A few more simple details about $(M, g)$ and the action above will be needed. Namely, let $C$ be the horizontal distribution orthogonal to $V$. Then, we can define the 2-tensor

$$
r(X, Y)=g\left(p r_{C} X, \operatorname{pr}_{C} Y\right)
$$

and the $s$-forms

$$
\omega^{u}\left(E_{v}\right)=\delta_{v}^{u}, \quad \omega^{u} / C=0 \quad(u, v=1, \ldots, s) .
$$

Since every vector field $\mathrm{X}$ has a unique decomposition

$$
\mathrm{x}=\mathrm{x}^{\prime}+\sum_{\mathrm{u}=1}^{\mathrm{s}} \omega^{\mathrm{u}}(\mathrm{X}) \mathrm{E}_{\mathrm{u}}, \quad \mathrm{X}^{\prime} \in \mathcal{C},
$$

we get

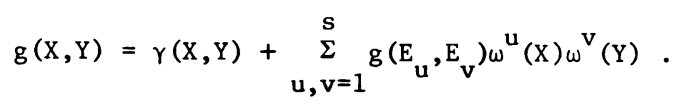

Furthermore since $E_{u}$ preserve $g$ and $V$, they also preserve $C$, and commute with $\mathrm{pr}_{\mathrm{C}}$. Hence

$$
\mathrm{L}_{\mathrm{E}}{ }_{\mathrm{u}}^{\gamma}=0, \quad \mathrm{~L}_{\mathrm{E}} \omega^{\mathrm{v}}=0
$$

Conversely, let $\left(M^{m}, \gamma, \omega^{u}\right) \quad(u=1, \ldots, s)$ be a differentiable manifold endowed with a positive semidefinite 2-covariant tensor $\gamma$ of rank $\mathrm{m}-\mathrm{s}$, and with $s$ independent Pfaff forms $\omega^{\mathbf{u}}$. Then $\omega^{\mathrm{u}}=0$ defines a subbundle $C$ of $T M$, and $\{X / i(X) \gamma=0\}$ defines a subbundle $V$. Assume that the structure is such that $T M=C \bullet V$. and 
define vector fields $E_{u}$ in $V$ such that $\omega^{v}\left(E_{u}\right)=\delta_{u}^{v}$. Furthermore, assume that the following relations hold

$$
\mathrm{L}_{\mathrm{E}} \gamma=0, \quad \mathrm{~L}_{\mathrm{E}} \omega^{\mathrm{v}}=0 .
$$

Then, we may define $g\left(E_{u}, E_{v}\right)=\delta_{u v}$, and use (4) in order to get a Riemannian metric admitting $E_{u}$ as Killing vector fields. Furthermore, we shall have

$$
\begin{aligned}
& \left(L_{E_{u}} \omega^{v}\right)\left(E_{w}\right)=0=-\omega^{v}\left(\left[E_{u}, E_{v}\right]\right), \\
& \left(L_{E_{u}}^{\gamma}\right)\left(E_{v}, X^{\prime}\right)=0=-\gamma\left(\left[E_{u}, E_{v}\right], X^{\prime}\right) \quad\left(X^{\prime} \in C\right),
\end{aligned}
$$

whence $\left[E_{u}, E_{v}\right]=0$ for all $u, v=1, \ldots, s$. Hence, if the structure $\left(\gamma, \omega^{u}\right)$ satisfies $T M=C \oplus V$ and (6) it provides $M$ with a Riemannian structure, and an isometric action of $\mathbb{R}^{S}$ with s-dimensiona1 orbits.

Now, we can formulate the following Boothby-Wang type fibration theorem

PROPOSITION. Let $\left(\mathrm{M}^{\mathrm{m}}, \mathrm{g}\right)$ be a compact connected Riemannian manifold endowed with a regular isometric action of $\mathbb{R}^{s}$ with the associated structure $\left(\gamma, \omega^{u}\right)$ defined above, and with the vertical foliation $V$. Then, the projection $\mathrm{p}: M \rightarrow B=M / V$ is a $T^{S}$ (torus)-principal bundle endowed with a connection $\left(c_{u} \omega_{u}\right)\left(c_{u}=\right.$ const.). The basis $B$ has a Riemannian metric $\gamma^{\prime}$ such that $p^{*} \gamma^{\prime}=\gamma$, and $p$ is a Riemannian submersion. Conversely, if $p: M \rightarrow B$ is a $T^{S}$-principal bundle over the Riemannian manifold $\left(B, \gamma^{\prime}\right)$, and $\left(\omega^{u}\right)$ is a connection of this bundle then $M$ admits a Riemannian metric and a regular isometric action of $\mathbb{R}^{S}$ with $s$-dimensional orbits such that $P$ is a Riemannian submersion.

PROOF. The proof of the existence of the principal bundle structure required is exactly the same as in the case of Proposition 3.1. All the other facts stated in Proposition are easy consequences of the formulas (1) - (6). R.E.D.

\section{REFERENCES}

1. LEE, H.C. A Kind of Even Dimensional Differential Geometry and its Application to Exterior Calculus, Amer. J. Math. 65 (1943), 433-438.

2. LIBERMANN, P. Sur les structures presque complexes et autres structures infinitésimales régulières, Bull. Soc. Math. France 83 (1955), 195-224.

3. LEFEBVRE, J. Transformations conformes et automorphismes de certaines structures presque symplectiques, C.R. Acad. Sc. Paris, t. 262 (1966), Série A. 752-754, and Propriétés du group des transformations conformes et du groupe des automorphismes d'une variété localement conformément symplectique, C.R. Acad. Sc. Paris, t. 268 (1969), Série A, 717-719.

4. VAISMAN, I. On Locally Conformal Almost Kähler Manifolds, Israel J. Math. 24 $(1976), 338-351$.

5. GRAY, A. and HERVELLA, L.M. The Sixteen Classes of Almost Hermitian Manifolds, Ann. Math. Pure Appl. 123 (1980), 35-58.

6. ABRAHAM, R. and MARDSEN, J. Foundation of Mechanics, 2nd Edit. Benjamin/Cummings Pub. Co. Reading Mass., 1978.

7. LICHNEROWICZ, A. Sur les algèbres de Kirillov-Shiga, C.R. Acad. Sc. Paris, t. 296 (1983), Série I, 915-920.

8. BOOTHBY, W.M. and WANG H.C. On Contact Manifolds, Ann. Math. 68 (1958), 721-734.

9. BLAIR, D.E., LUDDEN, G.D. and YANO, K. Differential Geometric Structures on Principal Toroidal Bundles, Trans. Amer. Math. Soc. 181 (1973), 175-184. 
10. DIAZ MIRANDA, A. and REVENTOS, A. Homogeneous Contact Compact Manifolds and Homogeneous Symplectic Manifolds, Bull. Science Math. 106 (1982), 337-350.

11. LICHNEROWICZ, A. Théorèmes de réductivité sur des algèbres d'automorphismes, Rendiconti di Mat. Roma 22 (1963), 197-244.

12. BLAIR, D.E. Geometry of Manifolds with Structural Group U(n) x $0(s)$, J. Diff. Geom. 4 (1970), 155-167.

13. TANNO, S. A Theorem on Regular Vector Fields and its Application to Almost Contact Structures, Tôhoku Math. J. 17 (1965), 235-243.

14. PALAIS, R. A Global Formulation of the Lie Theorie of Transformation Groups, Memoirs Amer. Math. Soc. 22, Providence, 1957. 


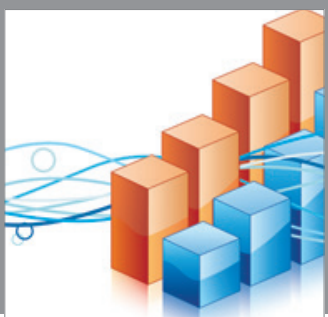

Advances in

Operations Research

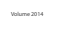

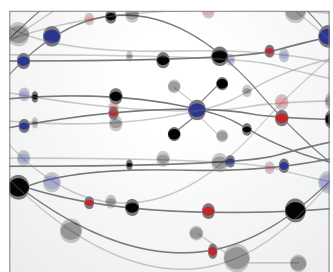

\section{The Scientific} World Journal
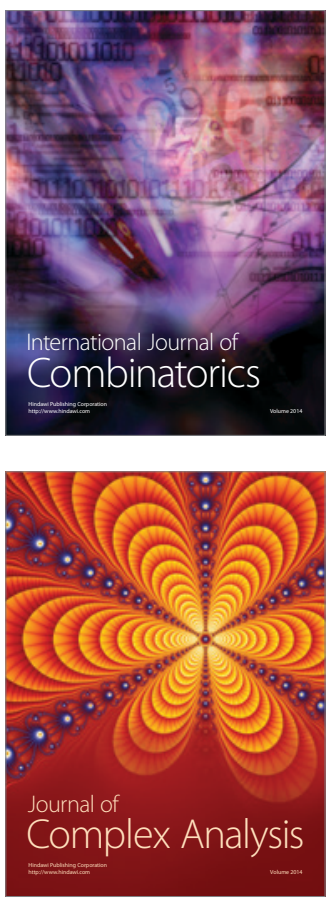

International Journal of

Mathematics and

Mathematical

Sciences
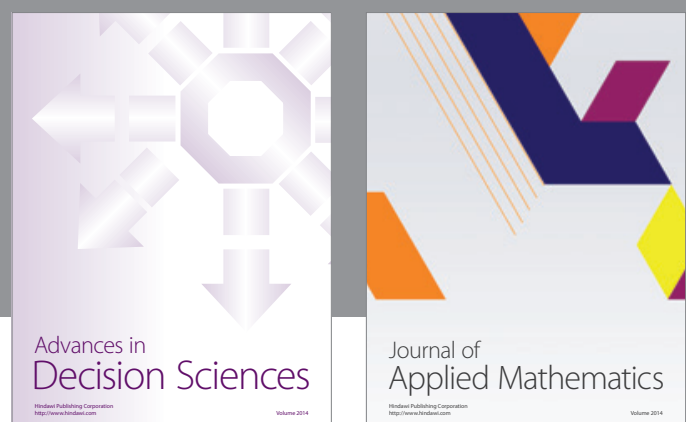

Journal of

Applied Mathematics
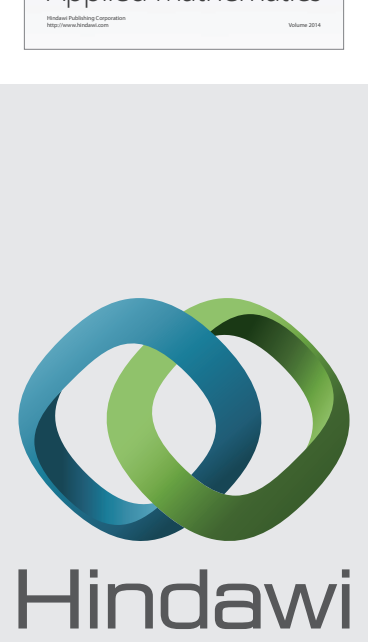

Submit your manuscripts at http://www.hindawi.com
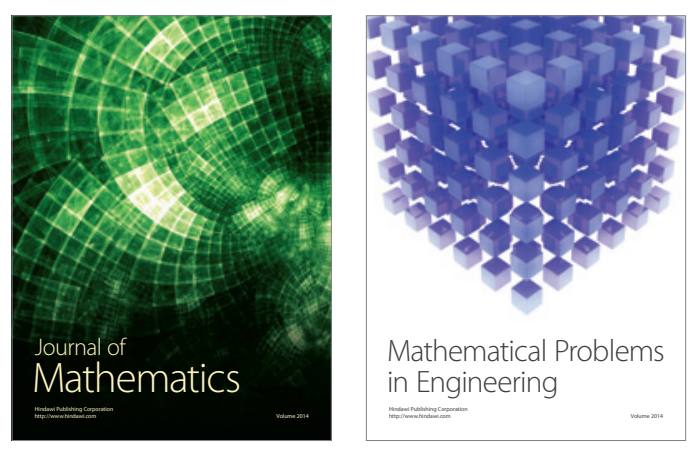

Mathematical Problems in Engineering
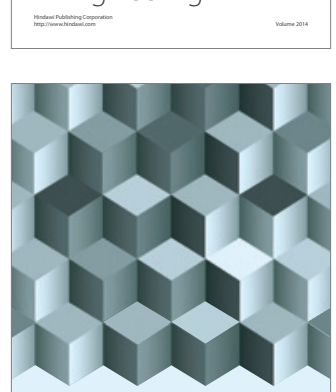

Journal of

Function Spaces
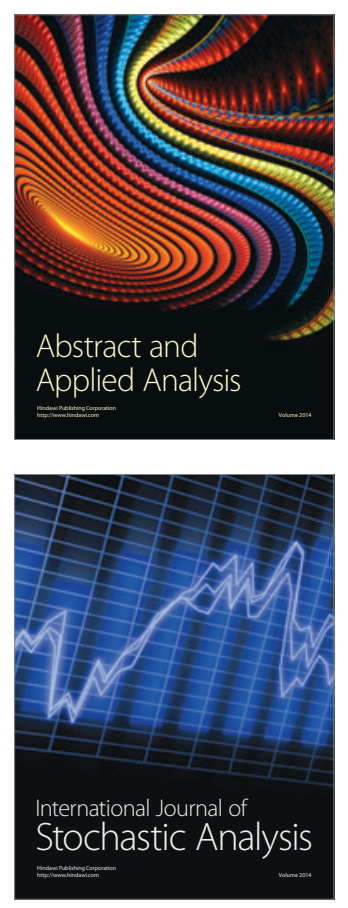

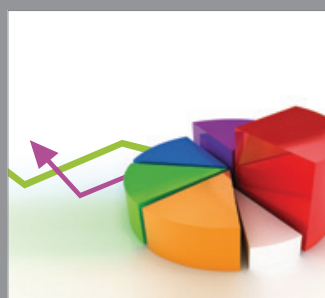

ournal of

Probability and Statistics

Promensencen
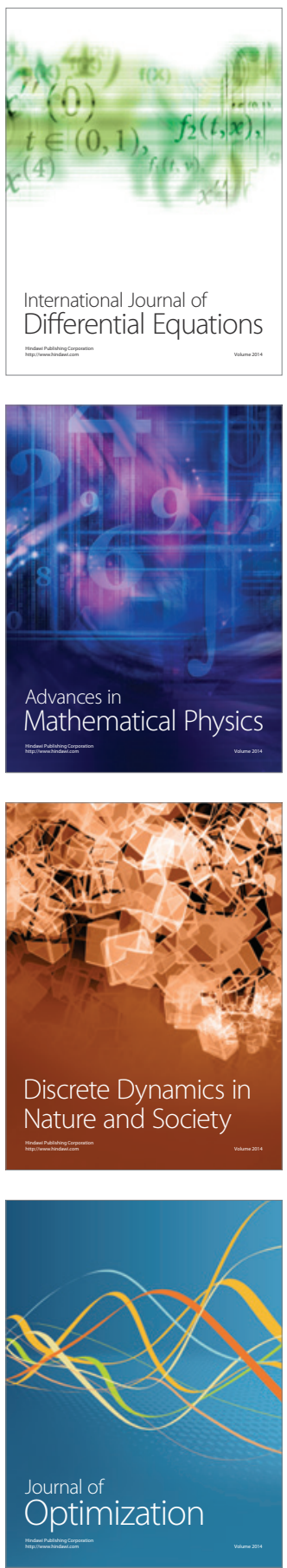